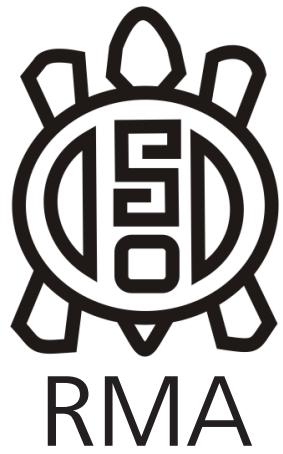

Dossier

\title{
Un abordaje del diseño, los gestos técnicos y las huellas de uso en instrumentos líticos experimentales (gubias, escoplos y cinceles), desde una aproximación funcional
}

\author{
A functional approach on the design, technical gestures and traces of \\ use in experimental lithic instruments (gouges, carvers and chisels)
}

Camila de Fátima Brizuela*

*Instituto de Antropología de Córdoba (IDACOR-CONICET), Museo de Antropología, Facultad de Filosofía y Humanidades, Universidad Nacional de Córdoba.

E-mail: cami.f.brizuela@gmail.com

\begin{abstract}
Resumen
Mediante un programa experimental y la metodología de análisis funcional de base microscópica como así también el estudio tipológico, este trabajo buscó entender los aspectos sociales que nos permitan reconocer y describir habitus o modos de hacer, focalizando en el estudio del diseño, los gestos técnicos y las huellas de uso que quedan registradas en las aristas activas y planos activos del instrumental lítico. Pretendiendo así, contribuir con la discusión de la relación entre diseño/forma y función de los instrumentos; centrando nuestro interés específicamente en tres grupos tipológicos definidos por Hocsman y Aschero (2015) como gubias, escoplos y cinceles. Puesto que los autores discuten aspectos vinculados con las "variables morfológico-funcionales", las "funciones primarias", su vinculación con "modos de acción" específicos y las "características de performance", entendíamos que era necesaria una caracterización y contrastación entre las huellas/gestos de uso y la variabilidad morfológica de estos grupos, buscando analizar las variables micro-morfológicas que los caracterizan y evaluar posibles similitudes y/o diferencias en sus contextos de uso y en las evidencias microscópicas de las áreas activas de estos instrumentos.
\end{abstract}

Palabras Claves: Modos de acción; Habitus; Grupos tipológicos; Variabilidad morfológica; Microscopía.

\begin{abstract}
Through the application of an experimental program and a methodology of microscopic based functional analysis as well as the typological study, this work sought to understand the social aspects that allow us to recognize and describe habitus or ways of doing, focusing on the study of design, technical gestures and the traces of use that are registered in the active edges and active planes of the lithic instruments. In this way, it aims to contribute to the discussion of the links between design/form and function of lithic instruments, specifically focusing our interest on the typological groups defined by Hocsman and Aschero (2015) as gouges, carvers and chisels. Since the authors discuss aspects related to the "morphological-functional variables", the "primary functions", their link with specific "modes of action" and "performance characteristics", we understood that a characterization and contrast between the traces / gestures of use and the morphological variability of these groups was necessary, seeking to analyze the micro-morphological variables that characterize them and evaluate possible similarities and I or differences in their contexts of use and in the microscopic evidences of the active areas of these instruments.
\end{abstract}

Keywords: Modes of action; Habitude; Typological groups; Morphological variability; Microscopy.

Comprender el gesto técnico con el que se fabricó y utilizó un instrumento lítico, es un desafío que se ha planteado la disciplina arqueológica tras la observación de las huellas que quedan remanentes en el instrumental líticos luego de su uso.

Mediante la ejecución de un programa experimental, buscamos en este trabajo discutir directamente la relación entre el diseño de un instrumento lítico y su funcionalidad dependiente o no, del mismo. Para ello sometimos a la experimentación a tres grupos tipológicos definidos por Hocsman y Aschero (2015) como Gubias, Escoplos y Cinceles, que diferían de acuerdo a sus ítems físicos macroscópicos. No obstante, desde una perspectiva antropológica se comprende que la tecnología lítica no está constituida simplemente por los ítems físicos de la cultura material, sino que considera también el comportamiento tecnológico y la acción humana (Ingold, 1988; Hocsman, 2006). Siempre que se entienda por tecnología a todo corpus de artefactos, comportamientos 
y conocimientos para crear y usar productos que es transmitido intergeneracionalmente, como así también, gestos técnicos que son aprendidos y expresados por los individuos en el curso de prácticas sociales (Schiffer y Skibo 1987).

Siguiendo la "historia de las herramientas" (sensu LeroiGourhan, 1971), el autor plantea que no puede negarse que hay útiles que son mejores que otros para realizar cierta actividad, podría hablarse aquí de "funciones primarias" en términos de Aschero (1975). Hocsman y Aschero (2015) exponen, que "hay una cuestión de eficacia, o mejor, de eficacia relativa, que debe ser puesta en consideración" (p.293).

Aquí entra en juego la cinemática, el saber cómo se utilizó cierto instrumento. Semenov, (1964) al igual que la mayoría de los investigadores de análisis funcional (Mansur 1983,1986; entre muchos otros), han afirmado que el tipo de movimiento empleado nos informa cómo es el proceso de transformación de la materia, la progresión tecnológica y la eficacia del trabajo. Marcel Mauss (1979) había ya destacado la importancia de las técnicas corporales aprendidas en el marco de cada cultura. El cuerpo es el primer instrumento del hombre, señala Mauss, o para no hablar de instrumento, refiere más bien al objeto y medio técnico más normal del hombre. Una "verdadera biología de la técnica" (Leroi-Gourhan, 1971). Siguiendo a Boëda (1997), todo instrumento es una entidad mixta; es tanto un objeto material y es también el resultado de un esquema, donde el gesto técnico es el elemento estructurante. Según Bourdieu (2007) podria tratarse de "hábitus", en el sentido que lo reconoce como "sistemas de disposiciones duraderas y transferibles, estructuras estructuradas predispuestas a funcionar como estructuras estructurantes..."

\section{Gubias, escoplos y cinceles}

Estos grupos se añaden a la lista tipológica "Ensayo para una clasificación morfológica de artefactos líticos" (Aschero, 1975, 1983), luego de que se da cuenta de su presencia en distintos sitios arqueológicos de diversas temporalidades (Hoscman y Aschero, 2015). Los mismos responden a la acción de desbaste, presente en el proceso técnico de trabajo de materiales como el hueso y la madera que son característicos de un amplio periodo de ocupación y por lo tanto entendemos que nuestros resultados pueden ser de interés para una amplia comunidad científica (Figura 1). Respecto a sus características morfológicas se presenta la siguiente tabla, tomada y modificada de Hocsman y Aschero 2015 (Tabla 1).

\section{Materiales y métodos}

La muestra analizada representa una colección de 96 instrumentos experimentales, gubias $(\mathrm{N}=32)$, escoplos $(\mathrm{N}=32)$ y cinceles $(\mathrm{N}=32)$. El número se debe a las diferentes variables adoptadas para el programa experimental y su posterior análisis. Estas son: la materia prima del instrumento, el género de quién ejecutó la acción de desbaste, el tiempo de uso de los instrumentos y los materiales sobre los cuales trabajó. Se utilizó cuarzo de Tanti, Córdoba y vulcanita variedad 1 de Antofagasta de la Sierra, Catamarca. Por otro lado, la mitad de la muestra fue empleada por individuos femeninos y la otra mitad por masculinos. En relación al tiempo de uso, 48 instrumentos se utilizaron durante 15 minutos, y los 48 restantes hasta que su filo se vio embotado y ya no respondía a su finalidad. Finalmente, los materiales trabajados fueron: vegetales leñosos duros y blandos, y huesos en estado fresco y seco.

\section{Programa Experimental}

Nuestro programa experimental constó, en principio, de la manufactura de los instrumentos, continuando con el uso y registro de los mismos. Luego de la limpieza de los filos activos, se procedió a realizar el correspondiente análisis bajo microscopio.

\section{Manufactura de los instrumentos}

Ambas materias primas fueron talladas unifacialmente bajo diversas técnicas de formatización. En cuanto a las gubias su confección en vulcanita fue por percusión y presión, utilizando percutor de leño y retocador de hueso. Las piezas de cuarzo, se realizaron solo por presión con retocador de hueso. Para los escoplos, la confección sobre vulcanita fue por percusión y presión,
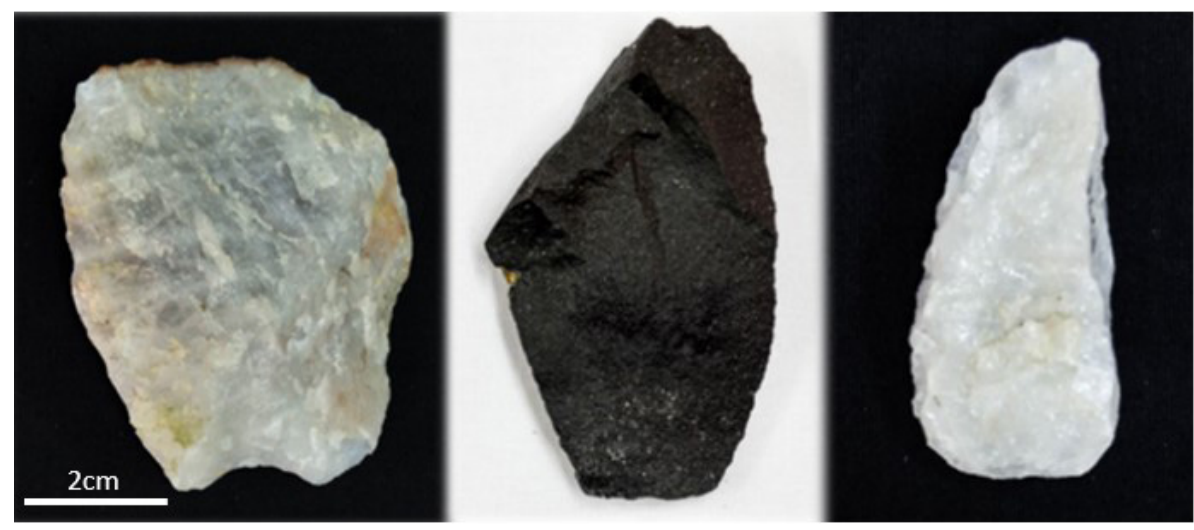

Figura 1. De izquierda a derecha: gubia, escoplo y cincel de nuestro programa experimental.

Figure 1. From left to right: gouges, carvers and chisels obtained by our experimental approach. 


\begin{tabular}{|c|c|c|c|}
\hline & GUBIAS & ESCOPLOS & CINCELES \\
\hline $\begin{array}{l}\text { Conformación del } \\
\text { borde }\end{array}$ & $\begin{array}{l}\text { Forma primaria: normal } \\
\text { regular }\end{array}$ & $\begin{array}{l}\text { Forma primaria: } \\
\text { normal regular }\end{array}$ & $\begin{array}{l}\text { Forma primaria: normal } \\
\text { regular }\end{array}$ \\
\hline $\begin{array}{l}\text { Delineación de la } \\
\text { arista }\end{array}$ & $\begin{array}{l}\text { Concavilínea atenuada, } \\
\text { media o semicircular }\end{array}$ & Rectilínea & Rectilínea \\
\hline $\begin{array}{c}\text { Forma geométrica } \\
\text { del filo }\end{array}$ & $\begin{array}{c}\text { Forma secundaria: } \\
\text { rectilínea a convexilínea } \\
\text { atenuada }\end{array}$ & $\begin{array}{l}\text { Forma secundaria: } \\
\text { rectilínea o rectilínea } \\
\text { convergente }\end{array}$ & $\begin{array}{c}\text { Forma secundaria: } \\
\text { rectilínea regular o } \\
\text { concavilínea muy atenuada } \\
\text { o convexilínea muy } \\
\text { atenuada }\end{array}$ \\
\hline $\begin{array}{c}\text { Extensión relativa } \\
\text { del filo }\end{array}$ & $\begin{array}{l}\text { Corto o restringido frontal, } \\
\text { transversal al eje mayor o } \\
\text { longitudinal al artefacto }\end{array}$ & $\begin{array}{l}\text { Corto o restringido } \\
\text { frontal, transversal al } \\
\text { eje longitudinal o al } \\
\text { eje mayor }\end{array}$ & $\begin{array}{l}\text { Corto o restringido frontal, } \\
\text { transversal al eje } \\
\text { longitudinal o al eje mayor }\end{array}$ \\
\hline Ángulo del bisel & Menor a $50^{\circ}$ & Mayor a $50^{\circ}$ & Mayor a $50^{\circ}$ \\
\hline Tamaño & $\begin{array}{l}\text { Mediano pequeño, } \\
\text { mediano grande o grande }\end{array}$ & $\begin{array}{c}\text { Mediano pequeño, } \\
\text { mediano grande o } \\
\text { grande }\end{array}$ & $\begin{array}{c}\text { Mediano pequeño, } \\
\text { mediano grande o grande }\end{array}$ \\
\hline $\begin{array}{l}\text { Módulo longitud- } \\
\text { anchura }\end{array}$ & $\begin{array}{l}\text { Mediano normal o } \\
\text { mediano alargado }\end{array}$ & $\begin{array}{l}\text { Mediano normal o } \\
\text { alargado o laminar } \\
\text { normal, angosto o } \\
\text { muy angosto }\end{array}$ & $\begin{array}{c}\text { Mediano normal o alargado } \\
\text { o laminar normal, angosto } \\
\text { o muy angosto }\end{array}$ \\
\hline
\end{tabular}

Tabla 1. Características morfológico-funcionales de las gubias, los escoplos y los cinceles (Adaptado de Hocsman y Aschero, 2015, p.289).

Table 1. Morphologicalfunctional features of the gouges, carvers and chisels (adapted from Hocsman and Aschero, 2015, p:289)

se utilizó un percutor de leño y un retocador de hueso. Mientras que para cuarzo, la confección fue también por percusión y presión, pero con percutor de piedra blanda y retocador de hueso. Finalmente, en cuanto a los cinceles, la técnica de talla sobre vulcanita fue por percusión y presión, se utilizó percutor y retocador de hueso. Para los instrumentos de cuarzo, se confeccionó solo uno por presión, nueve por percusión y seis por percusión y presión con percutor de piedra blanda y retocador de hueso.

Experiencias de uso de los instrumentos

El uso de cada instrumento se realizó por equipos de dos personas; mientras una de ellas realizaba la acción de desbaste, la otra registraba. A los fines de llevar un registro controlado de cada experiencia se generó una planilla donde se detalló en principio fecha de realización de la actividad, personas involucradas en la experiencia y el código predefinido del instrumento. Luego se detallaron todos aquellos atributos morfo-funcionales del instrumento y sus modos de uso, como así también las experiencias del ejecutante de la acción durante el transcurso de la actividad.

\section{Limpieza}

La limpieza de los residuos adheridos en la arista utilizada constó, en un primer raspado del material trabajado (con espátulas plásticas), adherido a la arista activa; guardando los residuos en tubos Eppendorf. Seguido por una limpieza más profunda para el posterior análisis bajo microscopio; empleando hisopo y acetona a través de pequeños movimientos circulares. Estos residuos formarían parte de una segunda colección de referencia que sería analizada por la Dra. Pilar Babot (ISES, CONICET-UNT - IAM, FCNeIML, UNT) en sus estudios comparativos de macro y microrestos.

\section{Análisis microscópico}

Para el análisis microscópico se aplicaron las propuestas desarrolladas por Semenov (1964), Keeley (1980), Mansur (1986, 1999), trabajados ampliamente en Argentina desde entonces (Álvarez et al., 2000; Álvarez, et al., 2001; Mansur y Lasa, 2005; Cattáneo y Fernández Ordoñez, 2005; Cattáneo, 2006; Flegenheimer y Leipus, 2007; Leipus y Mansur, 2007; Cueto, 2012; Cueto y Frank, 2010; Pal, 2015; De Angelis, 2013, 2016; entre otros).

Se llevó a cabo una aproximación óptica según tres niveles de observación, en un orden creciente de estudio diagnóstico de los rastros de uso. Se comenzó con Lupa Estereoscópica Binocular Motic (menos de 100X), se procedió al análisis con microscopio Metalográfico de reflexión Motic (entre 100 X y 500X), ambos instrumentos cuentan con cámaras de fotos integradas y, finalmente, se hizo uso del microscopio Láser Confocal Olympus 
LEXT (de 10X a más de 5000X) utilizando un software especial del equipo para la obtención de imágenes en formato tiff.

\section{Resultados}

\section{Gubias}

Respecto al modo de empleo de las gubias, el posicionar las piernas cruzadas, una de ellas flexionada y la otra estirada, o ambas piernas estiradas fueron algunas de las posiciones que se escogieron; como así también, aunque en menor medida, el direccionar la fuerza desde una posición más elevada, sentándose en un banco, por ejemplo. En todas las experiencias los ejecutantes optaron por inclinar su porción superior del cuerpo hacia adelante para aumentar así la presión ejercida.

En relación a la técnica de uso empleada, el 56\% de las experiencias se realizaron mediante presión ya sea interdigital (sujeción del instrumento entre dos o más dedos) y/o dígito-palmar (sujeción del instrumento entre los dedos y la palma de la mano), otro 38\% por percusión indirecta (en todos los casos de percusión se utilizó percutor de madera) y el $6 \%$ restante, combinando ambas. A nivel general los tramos de pasadas que el instrumento realizaba sobre el material trabajado fueron cortos y la fuerza aplicada aumentaba gradualmente.

Siguiendo el modo de empleo de las gubias bajo un ángulo de ataque bien abierto, las formas de sujetarla se fueron adecuando a las particularidades morfológicas del instrumento y del material trabajado. Por ello, los ejecutantes debían variar en ciertas ocasiones el ángulo, optando por cerrarlo moderadamente cuando la situación lo ameritaba; por ejemplo, al momento de retirar los nudos de un vegetal leñoso.

En cuanto a los resultados obtenidos mediante análisis funcional (Figura 2) y considerando las materias primas en las que fueron elaboradas, vemos que el desgaste por lo general fue lineal en zonas estrechas. La superficie se encuentra más estallada en los instrumentos de cuarzo y más homogénea en la vulcanita con algunos puntos estallados aislados que podrían responder a superficies de la matriz vítrea de esta variedad de la roca. Respecto al tiempo de uso, las experiencias de 15 minutos, se realizaron todas en ese tiempo. A pesar de encontrarse algunas resistencias durante la experimentación, se continuó a fin de confirmarse lo planteado de que un lapso corto de uso dificulta el análisis comparativo de rasgos diagnósticos (Álvarez, et al., 2001; De Angelis y Mansur, 2010). En cuanto al otro tiempo de uso hasta que el filo se embote, observamos que el promedio de tiempo de actividad de las gubias de cuarzo fue de $25^{\prime}$. Algunas experiencias concluyeron en los primeros minutos por motivo de estallado de la pieza frente a los golpes del percutor, por ejemplo, no por embotamiento, y eso dificultaba la continuidad del desbaste. No obstante, hubo casos de gubias de cuarzo que alcanzaron la hora de desbaste. En cuanto a las gubias de vulcanita predestinadas a trabajar hasta embotarse, se observaron tiempos mínimos de uso de $15^{\prime}$ y máximos de 52', con un promedio de 33' para que las gubias de vulcanita variedad 1 se emboten bajo las condiciones y variables consideradas en nuestro programa experimental. A diferencia de las gubias de cuarzo no hubo casos donde el filo estalle a los primeros minutos de actividad. Por su parte en una de las experiencias que alcanzó los 43 'de uso, el filo se reactivaba y ayudaba a prolongar el tiempo de actividad, pero a su vez las microlascas desprendidas puede que se llevasen consigo huellas de uso de momentos anteriores. En relación con el género de las personas involucradas en la actividad, se observó que el desgaste de las aristas activas fue parejo en ambos géneros. Cuando observamos diferencias en la intensidad de los rastros, pudimos atribuirlas al tiempo en que fue trabajado cada uno de los instrumentos. Finalmente, podemos decir que fue sobre los vegetales leñosos duros dónde el desgaste se percibe con mayor intensidad en relación a los vegetales leñosos de menor dureza observándose en ambos machacado suave inicial en zonas elevadas y desgaste ligeramente ondulado con posibles melladuras. En relación a los huesos, la diferencia fue más marcada. En aquellos en estado fresco el desgaste reflejaba un brillo mate ligeramente ondulado y suave; y en los de hueso seco hubo mayor estallamiento, con un fuerte brillo reflejado.

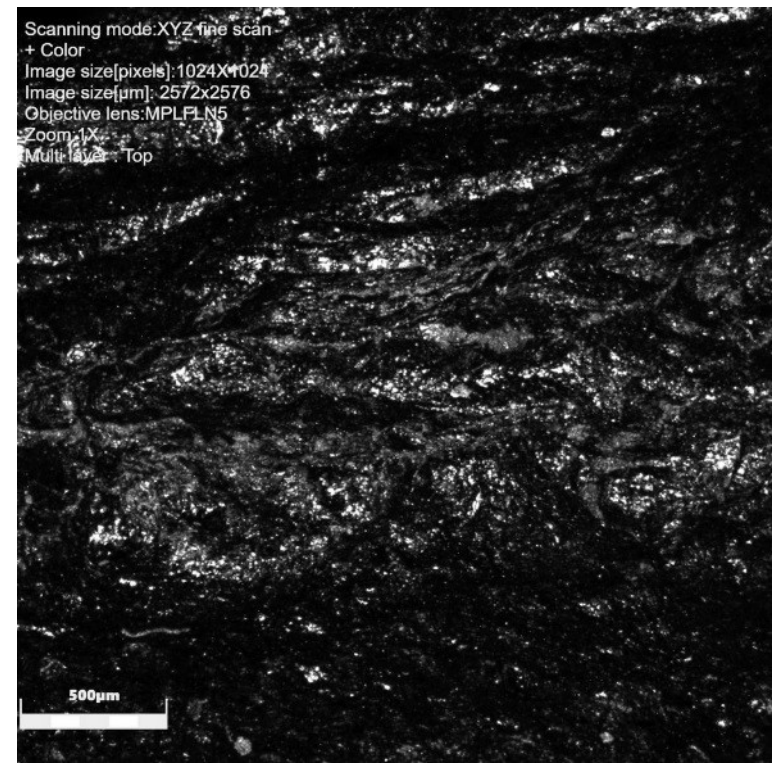

Figura 2. Micropulido observado bajo microscopio láser confocal LEXT (108X) de una gubia de vulcanita variedad 1 utilizada por un individuo masculino para desbastar hueso seco por 21 minutos.

Figure 2. Micro-wear seen low confocal laser microscope LEXT (108X) of a vulcanite gouge variety 1 used by a male individual to scrape bone for 21 minutes. 


\section{Escoplos}

En cuanto a los escoplos, las técnicas corporales de uso se asemejaron en gran medida a la de las gubias; y como destacamos con anterioridad, en todas las experiencias los ejecutantes optaron por inclinar su porción superior del cuerpo hacia adelante para aumentar así la presión ejercida. Respecto a la técnica de uso empleada, el escoplo se utiliza mediante presión y empuje, con prensión dígito-palmar o interdigital. No obstante, una de las experiencias (a modo de prueba) fue intercalando la técnica de presión con la de percusión indirecta durante toda la actividad; pero debido al ángulo obtuso del escoplo debía percutirse con gran fuerza alterando el gesto preestablecido. A nivel general, los tramos de pasadas que el instrumento realizaba sobre el material trabajado fueron largos y con respecto a la fuerza a un comienzo de las actividades era moderada y con el paso de los minutos por lo general aumentaba gradualmente compensando la pérdida de filo.

En cuanto al análisis microscópico (Figura 3) y considerando las materias primas, el desgaste aquí se observa en forma de campos y no en zonas estrechas. En los instrumentos de cuarzo, se distinguen estallados y superficies machacadas y posibles microlascados junto con micropulidos reflejándose un fuerte brillo producto del descabezamiento de las cimas. En los de vulcanita el machacado es más suave y homogéneo de textura más ondulada. En relación al tiempo de uso a los que

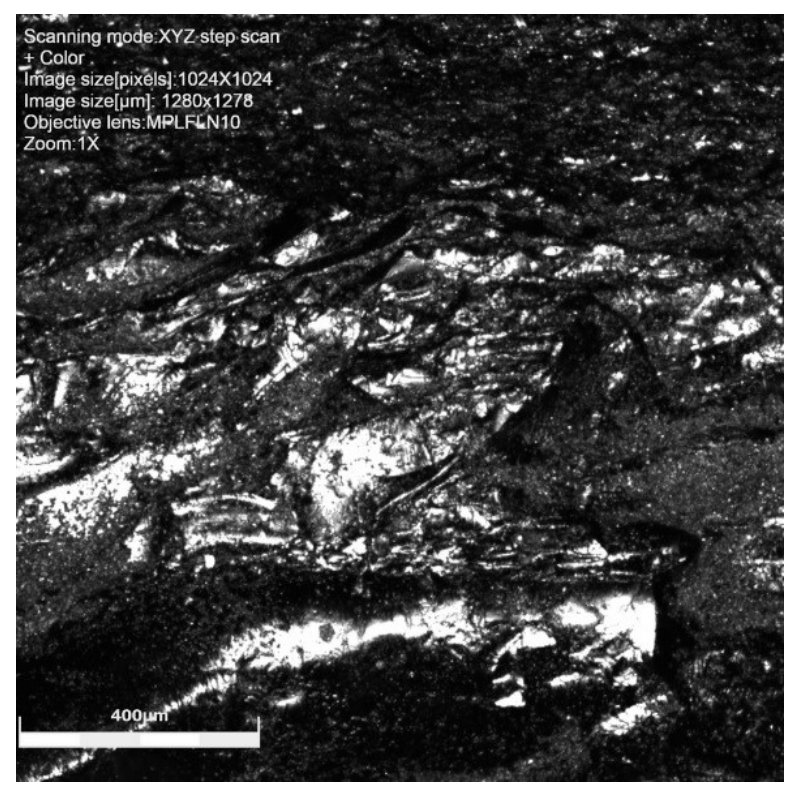

Figura 3. Micropulido intenso observado bajo microscopio láser confocal LEXT (216X) de un escoplo de cuarzo utilizado por un individuo masculino para desbastar vegetal leñoso blando por 77 minutos.

Figure 3. Intense micro-wear seen low confocal laser microscope LEXT $(216 X)$ of a quartz carvers used by a male individual to scrape soft woody vegetable for 77 minutes. se les preestableció un uso de 15 minutos, al igual que con las gubias, se decidió alcanzar dicho tiempo a pesar de los obstáculos que podían presentarse. Y en relación al otro tiempo de uso se observó que, en los escoplos de cuarzo, el promedio de vida útil del filo fue de 38', con experiencias que se embotaron a los 18' y otras que alcanzaron los 77'. Para el caso de las vulcanitas variedad $1,28^{\prime}$ fue el promedio de actividad, con tiempos que iban de 15' a 42'; destacando así, que es menor la vida útil de escoplos de Vc1 que el de Qz. Al igual que en las gubias, el género del ejecutante no fue una variable de gran relevancia ya que no se encontraron diferencias significativas entre individuos masculinos y femeninos. Finalmente, en cuanto a los materiales trabajados, por lo general en los vegetales leñosos la superficie se encuentra ligeramente desgastada con una suavidad de apariencia ondulada y estallada en sectores, reflejando así puntos aislados de fuerte brillo. Por su parte, en los instrumentos que trabajaron sobre hueso, se destaca el machacado y estallado más severo en los casos de hueso seco, reflejando mayor brillo y observándose mayor porcentaje de bordes afectados. En los casos de hueso fresco, el machacado está presente en menor medida, reflejando un brillo más opaco de apariencia ondulada.

\section{Cinceles}

En cuanto a los cinceles, sentarse en el suelo continuó siendo una de las posiciones más comunes con las piernas cruzadas o con una pierna estirada y la otra flexionada. Sin embargo, muchos ejecutantes optaron por realizar la actividad por encima del nivel del suelo, apoyando el material trabajado en una superficie elevada e inclinando la porción superior del cuerpo hacia adelante. En cuanto a la técnica de uso, el 25\% de las experiencias se emplearon a modo de prueba por presión mediante prensión interdigital o dígito-palmar y el 75\% restante por percusión indirecta. En este caso, por lo general, los primeros golpes sirvieron para acomodar el instrumento de una forma cómoda y lograr adquirir un gesto técnico de uso adecuado. Algunas tuvieron la participación de otra persona que facilitaba la sujeción del material trabajado. A nivel general los tramos de pasadas que el instrumento realizaba sobre el material trabajado fueron cortos y la fuerza aplicada continuaba siendo un factor que iba en aumento.

En cuanto a los rastros microscópicos (Figura 4) observamos que los micropulidos se presentan en campos como en escoplos. En la vulcanita se muestran suaves y con una textura más ondulada que en el cuarzo, donde es mayor el porcentaje de machacado y desgaste de bordes. Respecto al tiempo de uso de 15', todas las experiencias fueron realizadas con el fin de llegar a cumplir dicho tiempo preestablecido. Y para las experiencias cuyo tiempo de uso no se preestableció, en los cinceles de cuarzo el promedio de actividad del filo activo fue de 20', donde algunas de las experiencias concluyeron en los primeros minutos de actividad y otras alcanzaron los 


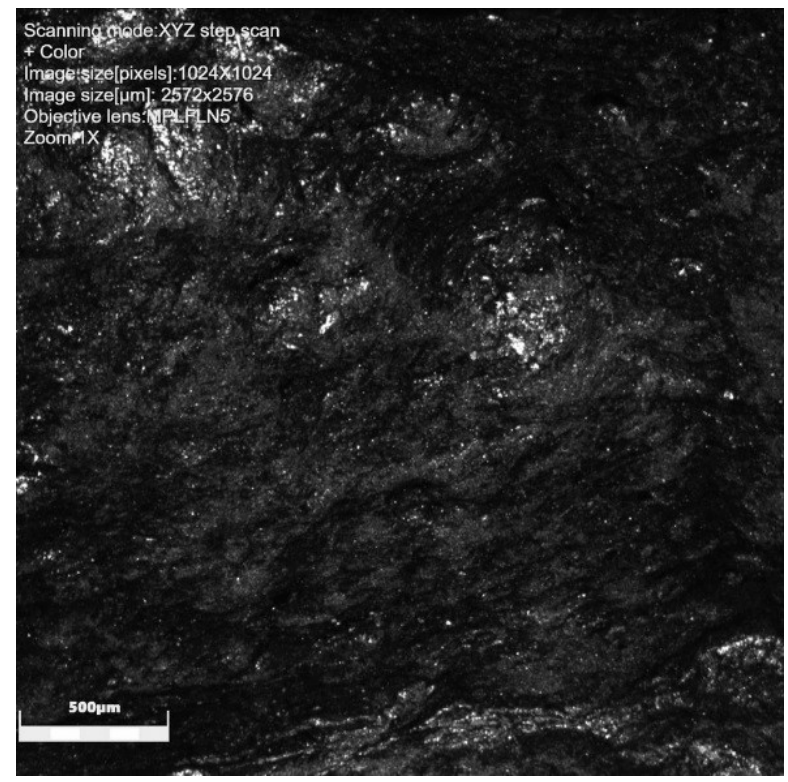

Figura 4. Micropulidos observados bajo microscopio láser confocal LEXT (108X) de un cincel de vulcanita variedad 1 utilizado por un individuo femenino para desbastar vegetal leñoso duro por 44 minutos.

Figure 4. Micro-wear seen low confocal laser microscope LEXT $(108 X)$ of a vulcanite chisel variety 1 used by a female individual to scrape hard woody vegetable for 44 minutes.

48'. Al igual que sucedía con algunas gubias de cuarzo trabajadas por percusión indirecta, la actividad concluía debido a la fractura de una porción del filo activo. En cuanto a los cinceles de vulcanita se observaron tiempos mínimos de $16^{\prime}$ y máximos de 53', arrojando esto un promedio de $28^{\prime}$ de vida útil. Al igual que en gubias y escoplos, tanto en individuos femeninos como en masculinos el desgaste está presente; y la diferencia de rastros es dependiente más bien de las demás variables. Finalmente, para los vegetales leñosos en sus distintos grados de dureza, está presente un machacado aislado o continuo de la superficie generando un brillo fuerte, como sucedía en el caso del trabajo sobre hueso seco. Para el trabajo sobre hueso fresco el desgaste se reflejaba en las superficies con un brillo más bien mate ligeramente ondulado, como percibimos también en gubias y escoplos.

\section{Discusión y Conclusiones}

En relación a nuestro objetivo de análisis de la relación entre diseño/forma y función, los instrumentos definidos en este caso como gubias, escoplos y cinceles fueron pensados y manufacturados para que cumplan con las variables que los caracterizan, detalladas en el artículo de Hocsman y Aschero (2015). Esto refiere a que antes de ser empleados en un trabajo específico, ya constituyen "diseños básicos". De este modo, una vez que son utilizados pueden sufrir modificaciones generándose así "diseños transformados", pero no en términos de
Aschero (1988), donde plantea que la transformación se da por mantenimiento o re-trabajado; sino que también, dichas transformaciones, pueden estar relacionados con el uso. Es muy común ver que en los primeros golpes el filo desprenda microlascas o se estalle levemente como parte de su reacción con la superficie de contacto. Aquí recae la importancia de evaluar cuáles pueden ser aquellos cambios que sufre el instrumento por el uso, por ejemplo, que la concavidad disminuya en las gubias y que haya pequeñas fracturas en los extremos, que hace que el filo se haga rectilíneo en norma sagital.

En algunas experiencias puntuales, quienes ejecutaron las acciones notaron que el desprendimiento progresivo de microlascas generaba reactivación del filo activo por el uso, o en términos de Mansur y Lasa (2005) "reavivado espontáneo del filo activo, ya que las propias microesquirlas arrastran consigo porciones del filo redondeado o alisado. Estas características son observables cuando se analiza el bisel con iluminación perpendicular y aumentos superiores a $200 X^{\prime \prime}$ (p.82). Este aspecto generaba prolongación, en la mayoría de los casos, de los minutos de actividad y facilitaba la penetración del instrumento en el material trabajado. No obstante, ésta reactivación implicaba cierta transformación que requería un cambio registrado en el modo de empleo del instrumento (entendiéndose por ejemplo, variaciones en el ángulo de contacto del instrumento lítico con el material trabajado). Siguiendo lo propuesto por Carlos Aschero (1975) y Daniele Lavallé, et al., (1995), se considera a los ángulos y la forma de los filos de los instrumentos como indicadores de funciones potenciales. Esta propuesta se basa en la hipótesis de que un ángulo de retoque de $50^{\circ}$, pertinente para una tarea, no serviría para cumplir la misma función que un ángulo de $80^{\circ}$, aunque posean la misma forma. Sin embargo, "la propuesta tipológica de Aschero (1975) considera e incluye categorías funcionales, basadas en la observación de filos a simple vista o con lupa para una atribución, pero con la salvedad de que estudios posteriores lo confirmen" (Cattáneo, 2006, p.34). Partiendo de dicha salvedad, exponemos aquí el caso particular de las gubias. Tal como describen Hocsman y Aschero (2015), el ángulo del bisel de este grupo tipológico es menor a $50^{\circ}$ y puede haber casos de reactivación del filo original donde el ángulo llegue a los $60^{\circ}$. Sin embargo, en nuestro programa experimental sólo 8 de las 32 gubias (25\% de las mismas) se confeccionaron con un bisel promedio cercano a los $50^{\circ}$. De aquí que creemos que ciertos factores como el ángulo de ataque del instrumento sobre el material trabajado o el gesto técnico aplicado para el uso pueden adecuarse a las particularidades morfológicas de un instrumento de desbaste logrando así, por ejemplo, que la gubia cuya arista activa posee $50^{\circ}$ y aquella de $80^{\circ}$ realicen la misma función. Entendemos que la escasa, o casi nula en algunos casos, diferenciación microscópica de rastros de uso entre los distintos grupos tipológicos, 
se debe en gran medida a que el ángulo del bisel de los instrumentos resultó ser muy similar en los tres grupos. Sin embargo, en general funcionaron adecuadamente en los contextos de uso propuestos, por lo que estaríamos frente a la situación de ampliar, en el "Ensayo para una clasificación morfológica de artefactos líticos aplicada a estudios tipológicos comparativos" (Aschero, 1975-83) y en la "Caracterización de los grupos tipológicos de las Gubias, los Escoplos y los Cinceles" (Hocsman y Aschero, 2015), los ángulos de los filos activos hasta incluso $80^{\circ}$, sin considerarlos filos embotados; o reconocer que esta modificación podría interpretarse desde el punto de vista morfológico como perteneciente a otro nuevo y distinto grupo tipológico. Creemos entonces que, es necesario continuar profundizando en estas características vinculadas a performances definidas, previo a establecer categorías fijas.

Córdoba, 25 de Enero 2019

\section{Agradecimientos}

A la Directora de este trabajo la Dra. Roxana Cattáneo y al Co-Director el Dr. Salomón Hocsman. Al proyecto Ongamira y sus miembros. Al Instituto de Antropología de Córdoba (IDACOR) y al Museo de Antropología. Al equipo de profesionales del Laboratorio de Microscopía Electrónica y Análisis por Rayos X (LAMARX) ubicado en la Facultad de Matemática, Astronomía y Física (FAMAF) de la Universidad Nacional de Córdoba, donde se tomaron las imágenes con Microscópio Láser Confocal Olympus LEXT. A mi familia y demás compañerxs y amigxs por su participación activa en el programa experimental presentado.

\section{Bibliografía}

Álvarez, M., Lasa, A. y Mansur, E. (2000). La explotación de recursos naturales perecederos. Análisis funcional de los raspadores de la costa norte del Canal de Beagle. Relaciones de la Sociedad Argentina de Antropología XXV (NS):275-296. Buenos Aires, Argentina.

Álvarez, M., Fioret, D., Favret, E., Castillo Guerra, R. (2001). "The Use of Lithic and Analysis of UseWear Traces in Experimental Tools Through Optical Microscopy and SEM". JAS 28, 457-464.

Aschero, C. (1975). Ensayo para una clasificación morfológica de artefactos líticos aplicada a estudios tipológicos comparativos. Informe al CONICET. Buenos Aires. Inédito.

Aschero, C. (1983). Modificaciones en Ensayo para una clasificación morfológica de artefactos líticos aplicada a estudios tipológicos comparativos. Apéndices A-C. Revisión. Cátedra de Ergología y Tecnología (FFyL-UBA). Buenos Aires. Inédito
Aschero, C. (1988). De punta a punta: producción, mantenimiento y diseño en puntas de proyectil precerámicas de la Puna argentina. Actas del IX Congreso Nacional de Arqueología Argentina, pp. 219-229. Buenos Aires.

Boëda, E. (1997). "Technogenèse de systèmes de production lithique au Paléolithique inférieur et moyen en Europe occidentale et au Proche-Orient". Habilitation à diriger des recherches Université de Paris-X-Nanterre.

Bourdieu, P. (2007). El sentido práctico. Siglo XXI Editores Argentina S. A. Traducido por Ariel Dilon / / ISBN 978987-1220-84-7

Cattáneo, R. G. (2006). Tecnología Lítica del Pleistoceno Final/Holoceno Medio. Un Estudio de los CazadoresRecolectores de la Patagonia Austral (Argentina). BAR Internacional Series1580. Oxford.

Cattáneo, R. G. y Fernández Ordoñez, M. (2005). Programa de observación de microhuellas de uso en instrumentos líticos y su aporte a la discusión de la funcionalidad de sitios: datos experimentales y arqueológicos. CONICET/ UNLP.

Cueto, M. (2012). Manufactura, utilización y análisis de huellas de uso sobre artefactos líticos tallados en rocas silíceas de la meseta central de santa cruz. Diseño y desarrollo del programa experimental. Entre Pasados y Presentes III 762-781.

Cueto, M. y Frank, A. (2010). Prueba experimental del trabajo del hueso con herramientas líticas. Tratamiento térmico y manifestación de trazas de uso. Patagonia, Argentina. Boletín de Arqueología Experimental 8.

De Angelis, H. (2013). Arqueología de la faja central de Tierra del Fuego. Una aproximación: Funcional-espacial. Tesis de Doctorado. Facultad de Ciencias Naturales y Museo, UNLP. Argentina. MS.

De Angelis, H. (2016). Cadenas operativas, materias primas y análisis tecno-funcional del conjunto lítico de Kami 1, Tierra del Fuego. En IX Jornadas de Arqueología de la Patagonia. Coyhaique, 20 al 25 de octubre de 2014. Pp. 167-178.

De Angelis, H. y Mansur, M. E. (2010). Artefactos de vidrio en contextos cazadores recolectores. Consideraciones a partir del análisis tecnológico y funcional. Revista Atlántica-Mediterránea de Prehistoria y Arqueología Social 12, pp. 59-73

Flegenheimer, N. y Leipus, M. (2007). Trabajar en un espacio reducido, Cerro el Sombrero, Abrigo 1, en Pacarina, Actas del XVII Congreso Nacional de 
Arqueología Argentina, San Salvador de Jujuy, pp. 141144.

Hayden, B., Franco, N. \& Stafford,J. (1996). Evaluating lithic strategies and design criteria. Stone Tools. Theoretical insights into human prehistory. G. H. Odell (ed.), pp. 9-45. Plenum press. New York.

Hocsman, S. (2006). "Producción Lítica, Variabilidad y Cambio en Antofagasta de la Sierra.-ca.5500-1500 AP-". Tesis para optar al grado académico de Doctor en Ciencias Naturales. P. (532).

Hocsman, S. y Aschero, C. (2015). Caracterización de los grupos tipológicos de las Gubias, los Escoplos y los Cinceles. Comechingonia. Revista de Arqueología. 19(2): 281-296.

Ingold, T. (1988). "Tools, minds and machines: an excursion in the philosophy of technology". Techniques et Culture 12: 151-176.

Keeley, L. H. (1980). "Experimental determination of stone tool use: a microwear analysis". University of Chicago Press. Chicago.

Lavallée, D., Julien, M., Wheeler, J. y Karlin, C. (1995). Telarmachay. Cazadores y pastores prehistóricos de los Andes. Instituto Francés de Estudios Andinos, Tomo 88. Lima, Perú.

Leipus, M. y Mansur, M. E. (2007). «El análisis funcional de base microscópica aplicado a materiales heterogéneos. Perspectivas metodológicas para el estudio de las cuarcitas de la Región Pampeana», en Arqueología en las Pampas, Cristina Bayón, et al., eds., pp. 179-200. Buenos Aires: Sociedad Argentina de Antropología.

Leroi-Gourhan, A. (1971). "El gesto y la palabra".
Traducción: Felipe Carrera D. Ediciones de la biblioteca. Universidad Central de Venezuela.

Mansur-Franchomme, M. E. (1983).Traces d'utilisation et technologielithique: exemples de la Patagonie. Tesis de Doctorado. Institut du Quaternarie, Université de Bordeauxl.infor. Bâtgéologie, Bordeaux.

Mansur-Franchomme, M. E. (1986). "Microscopie du matériel lithique: traces d'utilisation, altérations naturelles, accidentelles et technologiques. Exemples de Patagonie". Cahiers du Quaternaire IX.

Mansur, M. E. (1999). "Análisis funcional de instrumental lítico: problemás de formación y deformación de rastros de uso". En Actas del XII Congreso Nacional de Arqueología Argentina. La Plata, pp. 355-366.

Mansur, M. E. y Lasa, A. (2005). Diversidad artefactual vs. Especialización funcional. Análisis del IV componente de túnel I (tierra del fuego, argentina). Magallania 33(2):6991.

Mauss, M. (1979 [1971]). Sociología y Antropología. Editorial Tecnos, S. A.

Pal, N. (2015). Identificación y caracterización de rastros de uso: programa experimental sobre rocas cuarcíticas y ftanita (provincia de Buenos Aires, Argentina). Boletín de Arqueología Experimental, 10: 161-173.

Schiffer, M. B. \& Skibo, J. M. (1987). Theory and Experiment in the Study of Technological Change. Current Anthropology 28: 595-622.

Semenov, S. A. (1964). Prehistoric Technology; an Experimental Study of the Oldest Tools and Artefacts from Traces of Manufacture and Wear. Cory, Adams \& Mackay, London. 